\title{
Stability and Stabilization of Continuous-Time Markovian Jump Singular Systems with Partly Known Transition Probabilities
}

\author{
Jumei Wei and Rui Ma \\ School of Mathematics and Statistics, Zhengzhou University, Zhengzhou 450001, China \\ Correspondence should be addressed to Jumei Wei; weijumei@zzu.edu.cn
}

Received 24 November 2013; Revised 27 February 2014; Accepted 27 February 2014; Published 3 April 2014

Academic Editor: Xiaojie Su

Copyright ( 92014 J. Wei and R. Ma. This is an open access article distributed under the Creative Commons Attribution License, which permits unrestricted use, distribution, and reproduction in any medium, provided the original work is properly cited.

\begin{abstract}
This paper investigates the problem of the stability and stabilization of continuous-time Markovian jump singular systems with partial information on transition probabilities. A new stability criterion which is necessary and sufficient is obtained for these systems. Furthermore, sufficient conditions for the state feedback controller design are derived in terms of linear matrix inequalities. Finally, numerical examples are given to illustrate the effectiveness of the proposed methods.
\end{abstract}

\section{Introduction}

In practice, many dynamical systems cannot be represented by the class of linear time-invariant model since the dynamics of these systems are random with some features, for example, abrupt changes, breakdowns of components, changes in the interconnections of subsystems, and so forth. Such class of dynamical systems can be adequately described by the class of stochastic hybrid systems. A special class of hybrid systems referred to as Markovian jump systems (MJS), a class of multimodel systems in which the transitions among different modes are governed by a Markov chain, have attracted a lot of researchers and many problems have been solved, such as stability, stabilization, and $H_{\infty}$ control problems; see [1-7].

However, in most of the studies, complete knowledge of the mode transitions is required as a prerequisite for analysis and synthesis of MJS. This means that the transition probabilities of the underlying Markov chain are assumed to be completely known. However, in practice, incomplete transition probabilities are often encountered especially if adequate samples of the transitions are costly or time consuming to obtain. So, it is necessary to further consider more general jump systems with partial information on transition probabilities. The concept for MJS with partially unknown transition probabilities is first proposed in [8] and a series of studies have been carried out [9-12] recently. A new approach for the analysis and synthesis for Markov jump linear systems with incomplete transition descriptions has been proposed in [12], which can be further used for other analysis and synthesis issues, such as the stability of Markovian jump singular systems (MJSS).

A lot of attention has already been focused on robust stability, robust stabilization, and $H_{\infty}$ control problems for MJSS in recent years, such as the works in [13-17]. However, to the best of the authors' knowledge, the necessary and sufficient conditions for the stochastic stability and stabilization problems of MJSS have not been fully investigated, especially when the transition probabilities are partially known. The authors in $[15,16]$ have, respectively, studied the problems of stability and stabilization for a class of continuous-time (discrete-time) singular hybrid systems. New sufficient and necessary conditions for these singular hybrid systems to be regular, impulse-free (causal), and stochastically stable have been proposed in terms of a set of coupled strict linear matrix inequalities (LMIs). But the case of systems with partly known transition probabilities still needs to be considered. In addition to this, it is important to mention that the derivation of strict LMIs for MJSS with incomplete transition probabilities renders the synthesis of the state feedback controllers easier. These problems are important and challenging in both theory and practice, which motivates us for this study.

In this paper, the problem of the stability and stabilization of MJSS with partly known transition probabilities is 
addressed. Inspired by the ideas in [12], which fully unitized the properties of the transition rate matrix (TRM) and the convexity of the uncertain domains, we explore a new sufficient and necessary condition in terms of strict linear matrix inequalities (LMIs) for the MJSS to be regular, impulsive, and stochastically stable. Then, based on the proposed stability criterion, the conditions for state feedback controller are derived. Finally, numerical examples are given to illustrate the effectiveness of the proposed method.

Compared with the existing works about the stability and stabilization of Markovian jump systems, the current paper has the following novel features. First, the current paper deals with the stability and stabilization problems for MJSS with partly known transition probabilities, while most literatures (e.g., [8-12]) focused on those of normal ones that are special cases of MJSS. Second, the conservatism in the conventional studies [15] is eliminated by considering the fact that the unknown elements of each row in TRM exist. Moreover, the difficulty that the unknown elements contain diagonal elements is also overcome by introducing a lower bound of the diagonal element without additional conservatism.

Notation. The notation used in this technical note is standard. The superscript " $T$ " stands for matrix transposition; $\mathbb{R}^{n}$ denotes the $n$ dimensional Euclidean space; $\mathbb{Z}^{+}$represents the sets of positive integers, respectively. For the notation $(\Omega, \mathscr{F}, \mathscr{P}), \Omega$ represents the sample space, $\mathscr{F}$ is the $\sigma$-algebra of subsets of the sample space, and $\mathscr{P}$ is the probability measure on $\mathscr{F}$. E[·] stands for the mathematical expectation. In addition, in symmetric block matrices or long matrix expressions, we use $*$ as an ellipsis for the terms that are introduced by symmetry and $\operatorname{diag}\left\{X_{1}, X_{2}, \ldots, X_{N}\right\}$ stands for a block-diagonal matrix constituted by $X_{1}, X_{2}, \ldots, X_{N}$. The notation $X>0$ means $X$ is real symmetric positive definite, and $X_{i}$ is adopted to denote $X(i)$ for brevity. $I$ and 0 represent, respectively, identity matrix and zero matrix. Matrices, if their dimensions are not explicitly stated, are assumed to be compatible for algebraic operations.

\section{Preliminaries and Problem Formulation}

Consider the following continuous-time MJSS with Markovian jump parameters:

$$
E \dot{x}(t)=A\left(r_{t}\right) x(t)+B\left(r_{t}\right) u(t)
$$

where $x(t) \in \mathbb{R}^{n}$ is the state vector and $u(t) \in \mathbb{R}^{m}$ is the control input. The matrix $E \in \mathbb{R}^{n \times n}$ is supposed to be singular with $\operatorname{rank}(E)=r<n$. The stochastic process $\left\{r_{t}, t \geq 0\right\}$ taking values in a finite set $S=\{1,2, \ldots, N\}$ is described by a continuous-time, discrete-state homogeneous Markov process and has the following mode transition probabilities:

$$
\operatorname{Pr}\left\{r_{t+h}=j \mid r_{t}=i\right\}= \begin{cases}\lambda_{i j} h+o(h), & \text { if } j \neq i, \\ 1+\lambda_{i i} h+o(h), & \text { if } j=i\end{cases}
$$

where $h>0, \lim _{h \rightarrow 0}(o(h) / h)=0$, and $\lambda_{i j} \geq 0(i, j \in S, j \neq i)$ denotes the switching rate from mode $i$ at time $t$ to mode $j$ at time $t+h$, and $\lambda_{i i}=-\sum_{j \in S, j \neq i} \lambda_{i j}$ for all $i \in S$. The TRM is given by

$$
\Lambda=\left[\begin{array}{cccc}
\lambda_{11} & \lambda_{12} & \cdots & \lambda_{1 N} \\
\lambda_{21} & \lambda_{22} & \cdots & \lambda_{2 N} \\
\vdots & \vdots & \ddots & \vdots \\
\lambda_{N 1} & \lambda_{N 2} & \cdots & \lambda_{N N}
\end{array}\right]
$$

The set $S$ contains $N$ modes of system (1) and for $r_{t}=$ $i \in S$, the system matrices of the $i$ th mode are denoted by $A_{i}, B_{i}$, which are known real-valued constant matrices of appropriate dimensions that describe the nominal system.

The transition rates described above are considered to be partially available; that is, some elements in matrix $\Lambda$ are unknown. Take system (1) with 4 operation modes for example; the TRM $\Lambda$ may be written as

$$
\Lambda=\left[\begin{array}{llll}
\lambda_{11} & \lambda_{12} & \hat{\lambda}_{13} & \hat{\lambda}_{14} \\
\hat{\lambda}_{21} & \hat{\lambda}_{22} & \lambda_{23} & \lambda_{24} \\
\hat{\lambda}_{31} & \widehat{\lambda}_{32} & \lambda_{33} & \lambda_{34} \\
\lambda_{41} & \lambda_{42} & \hat{\lambda}_{43} & \lambda_{44}
\end{array}\right],
$$

where “" denotes the unknown element.

For $\forall i \in S$, we denote

$$
S=S_{\mathscr{K}}^{i}+S_{\mathscr{K} \mathscr{K}}^{i}
$$

$S_{\mathscr{K}}^{i} \triangleq\left\{j: \lambda_{i j}\right.$ is known $\}, \quad S_{\mathscr{U} \mathscr{K}}^{i} \triangleq\left\{j: \lambda_{i j}\right.$ is unknown $\}$.

If $S_{\mathscr{K}}^{i} \neq \emptyset, S_{\mathscr{K}}^{i}$ is further described as

$$
S_{\mathscr{K}}^{i}=\left\{\mathscr{K}_{1}^{i}, \mathscr{K}_{2}^{i}, \ldots, \mathscr{K}_{m}^{i}\right\}, \quad 1 \leq m \leq N
$$

where $\mathscr{K}_{m}^{i} \in \mathbb{Z}^{+}$represents the index of the $m$ th known element in the $i$ th row of matrix $\Lambda$. Also, throughout the technical note, we denote

$$
\lambda_{\mathscr{K}}^{i}=\sum_{j \in S_{\mathscr{K}}^{i}} \lambda_{i j}
$$

When $\hat{\lambda}_{i i}$ is unknown, it is necessary to provide a lower bound $\lambda_{d}^{i}$ for it and $\lambda_{d}^{i} \leq-\lambda_{\mathscr{K}}^{i}$.

Now, we introduce the following definition for the continuous-time MJSS (1) (with $u(t) \equiv 0)$.

Definition 1 (see [17]).

(i) The continuous-time MJSS in (1) is said to be regular if, for each $i \in S, \operatorname{det}\left(s E-A_{i}\right)$ is not identically zero.

(ii) The continuous-time MJSS in (1) is said to be impulsive if, for each $i \in S, \operatorname{deg}\left(\operatorname{det}\left(s E-A_{i}\right)\right)=\operatorname{rank}(E)$.

(iii) The continuous-time MJSS in (1) is said to be stochastically stable if, for any $x_{0} \in \mathbb{R}^{n}$ and $r_{0} \in S$, there exists a scalar $M\left(x_{0}, r_{0}\right)>0$ such that

$$
\mathbf{E}\left\{\int_{0}^{\infty}\|x(t)\|^{2} \mid x_{0}, r_{0}\right\} \leq M\left(x_{0}, r_{0}\right),
$$

where $\mathbf{E}$ is the mathematical expectation, and $x\left(t, x_{0}, r_{0}\right)$ denotes the solution to system (1) at time $t$ under the initial conditions $x_{0}$ and $r_{0}$. 
(iv) The continuous-time MJSS in (1) is said to be stochastically admissible if it is regular, impulsive, and stochastically stable.

The following lemma is recalled, which will be used in what follows.

Lemma 2 (see [18]). Let $P \in R^{n \times n}$ be symmetric such that $E_{R}^{T} P E_{R}>0, \Phi \in R^{n \times n}$, and $S$ are nonsingular. Then, $P E+$ $S^{T} \Phi R^{T}$ is nonsingular and its inverse is expressed as

$$
\left(P E+S^{T} \Phi R^{T}\right)^{-1}=\bar{P} E^{T}+R \bar{\Phi} S,
$$

where $E_{L}$ and $E_{R}$ are full column rank with $E=E_{L} E_{R}^{T}, R \in$ $R^{(n-r) \times n}$, and $S \in R^{n \times(n-r)}$ satisfies $R E=0$ and $E S=0$, respectively. $\bar{P}$ is symmetric and $S$ is nonsingular such that

$$
\begin{gathered}
E_{L}^{T} \bar{P} E_{L}=\left(E_{R}^{T} P E_{R}\right)^{-1}, \\
\bar{\Phi}=\left(R R^{T}\right)^{-1} \Phi^{-1}\left(S S^{T}\right)^{-1} .
\end{gathered}
$$

\section{Main Results}

In this section, we will derive the stochastic stability criteria for system (1) when the transition probabilities are partially unknown and design a state-feedback controller and a static output feedback controller such that the closed-loop system is stochastically stabilizable. The mode-dependent controller considered here has the form

$$
u(t)=K\left(r_{t}\right) x(t),
$$

where $K_{i}=K\left(r_{t}\right) \in R^{m \times n}\left(\forall r_{t}=i \in S\right)$ are the controller gains to be determined. The closed-loop systems obtained by applying controllers (11) to system (1) are

$$
E \dot{x}(t)=\left(A_{i}+B_{i} K_{i}\right) x(t) .
$$

First, we provide the following lemma which presents a necessary and sufficient condition for the continuous-time MJSS with completely known transition probabilities matrix to be stochastically admissible.

Lemma 3 (see [15]). System (1) with $u(t)=0$ is stochastically admissible if and only if there exist matrices $P_{i} \in R^{n \times n}>0$, $i \in S$, and $\Phi_{i} \in R^{(n-r) \times(n-r)}$, such that the following coupled LMIs hold for each $i \in S$ :

$$
\begin{aligned}
& A_{i}^{T}\left(P_{i} E+R^{T} \Phi_{i} S^{T}\right)+\left(P_{i} E+R^{T} \Phi_{i} S^{T}\right)^{T} A_{i} \\
& \quad+\sum_{j \in S} \lambda_{i j} E^{T} P_{j} E<0 .
\end{aligned}
$$

Let us first give the stability result for the unforced system (1) (with $u(t) \equiv 0$ ). The following theorem presents a necessary and sufficient condition on the stochastic admissibility of the considered system with partially unknown transition probabilities.
Theorem 4. Consider the unforced system (1) with partially unknown transition probabilities. The corresponding system is stochastically admissible if and only if there exist matrices $P_{i} \in \mathbb{R}^{n \times n}>0$ and nonsingular symmetric matrices $\Phi_{i} \in$ $\mathbb{R}^{(n-r)(n-r)}$, such that for each $i \in S$

$$
\begin{gathered}
A_{i}^{T}\left(P_{i} E+R^{T} \Phi_{i} S^{T}\right)+\left(P_{i} E+R^{T} \Phi_{i} S^{T}\right)^{T} A_{i} \\
+E^{T} \mathscr{P}_{\mathscr{K}}^{i} E-\lambda_{\mathscr{K}}^{i} E^{T} P_{j} E<0, \\
\forall j \in S_{\mathscr{U} \mathscr{K}}^{i}, \text { if } i \in S_{\mathscr{K}}^{i}, \\
A_{i}^{T}\left(P_{i} E+R^{T} \Phi_{i} S^{T}\right)+\left(P_{i} E+R^{T} \Phi_{i} S^{T}\right)^{T} A_{i} \\
+E^{T} \mathscr{P}_{\mathscr{K}}^{i} E+E^{T}\left(\lambda_{d}^{i} P_{i}-\lambda_{d}^{i} P_{j}-\lambda_{\mathscr{K}}^{i} P_{j}\right) E<0, \\
\forall j \in S_{\mathscr{U} \mathscr{K}}^{i}, \text { if } i \in S_{\mathscr{U} \mathscr{K}}^{i},
\end{gathered}
$$

where $\mathscr{P}_{\mathscr{K}}^{i}=\sum_{j \in S_{\mathscr{K}}^{i}} \lambda_{i j} P_{j}$ and $\lambda_{d}^{i}$ is a given lower bound for the unknown diagonal element.

Proof. Consider two cases, $i \in S_{\mathscr{K}}^{i}$ and $i \in S_{\mathscr{K} \mathscr{K}}^{i}$, and note that system (1) is stochastically stable if and only if (13) holds.

Case $1\left(i \in S_{\mathscr{K}}^{i}\right)$. It should be noted that in this case one has $\lambda_{\mathscr{K}}^{i} \leq 0$. We only need to consider $\lambda_{\mathscr{K}}^{i}<0$ since $\lambda_{\mathscr{K}}^{i}=0$ means the elements in the $i$ th row of the TRM are known, so it is not considered here. Now the left-hand side of (13) in Lemma 3 can be rewritten as

$$
\begin{aligned}
\Theta_{i} \triangleq & A_{i}^{T}\left(P_{i} E+R^{T} \Phi_{i} S^{T}\right)+\left(P_{i} E+R^{T} \Phi_{i} S^{T}\right)^{T} A_{i} \\
& +\sum_{j \in S_{\mathscr{H}}^{i}} \lambda_{i j} E^{T} P_{j} E+\sum_{j \in S_{\mathscr{H}}^{i}} \widehat{\lambda}_{i j} E^{T} P_{j} E \\
= & A_{i}^{T}\left(P_{i} E+R^{T} \Phi_{i} S^{T}\right)+\left(P_{i} E+R^{T} \Phi_{i} S^{T}\right)^{T} A_{i} \\
& +E^{T} \mathscr{P}_{\mathscr{K}}^{i} E-\lambda_{\mathscr{K}}^{i} \sum_{j \in S_{\mathscr{H C H}}^{i}} \frac{\widehat{\lambda}_{i j}}{-\lambda_{\mathscr{K}}^{i}} E^{T} P_{j} E,
\end{aligned}
$$

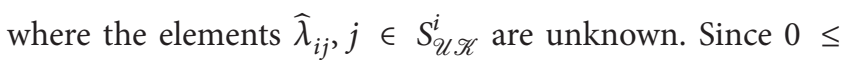
$\widehat{\lambda}_{i j} /\left(-\lambda_{\mathscr{K}}^{i}\right) \leq 1$ and $\sum_{j \in S_{\mathscr{H}}^{i}} \widehat{\lambda}_{i j} /\left(-\lambda_{\mathscr{K}}^{i}\right)=1$, we know that

$$
\begin{aligned}
& \Theta_{i}=\sum_{j \in S_{U \mathscr{K}}^{i}} \frac{\hat{\lambda}_{i j}}{-\lambda_{\mathscr{K}}^{i}} \\
& \times\left[A_{i}^{T}\left(P_{i} E+R^{T} \Phi_{i} S^{T}\right)+\left(P_{i} E+R^{T} \Phi_{i} S^{T}\right)^{T} A_{i}\right. \\
& \left.+E^{T} \mathscr{P}_{\mathscr{K}}^{i} E-\lambda_{\mathscr{K}}^{i} E^{T} P_{j} E\right] .
\end{aligned}
$$

Therefore, for $0 \leq \hat{\lambda}_{i j} \leq-\lambda_{\mathscr{K}}^{i}, \Theta_{i}<0$ is equivalent to $A_{i}^{T}\left(P_{i} E+\right.$ $\left.R^{T} \Phi_{i} S^{T}\right)+\left(P_{i} E+R^{T} \Phi_{i} S^{T}\right)^{T} A_{i}+E^{T} \mathscr{P}_{\mathscr{K}}^{i} E-\lambda_{\mathscr{K}}^{i} E^{T} P_{j} E<$ $0, \forall j \in S_{\mathscr{C} K}^{i}$, which implies that, in the presence of unknown 
elements $\hat{\lambda}_{i j}$, the system stochastic admissibility is ensured if and only if (14) holds.

Case $2\left(i \in S_{\mathscr{U}}^{i}\right)$. In this case, $\hat{\lambda}_{i i}$ is unknown, $\lambda_{\mathscr{K}}^{i} \geq 0$, and $\hat{\lambda}_{i i} \leq-\lambda_{\mathscr{K}}^{i}$. We also only consider $\widehat{\lambda}_{i i}<-\lambda_{\mathscr{K}}^{i}$ since $\widehat{\lambda}_{i i}=-\lambda_{\mathscr{K}}^{i}$; then the $i$ th row of the TRM is completely known.

Now the left-hand side of (15) can be rewritten as

$$
\begin{aligned}
& \Theta_{i} \triangleq A_{i}^{T}\left(P_{i} E+R^{T} \Phi_{i} S^{T}\right)+\left(P_{i} E+R^{T} \Phi_{i} S^{T}\right)^{T} A_{i} \\
& +E^{T} \mathscr{P}_{\mathscr{K}}^{i} E+\widehat{\lambda}_{i i} E^{T} P_{i} E+\sum_{j \in S_{\ell \mathscr{K}}^{i}, j \neq i} \hat{\lambda}_{i j} E^{T} P_{j} E \\
& =A_{i}^{T}\left(P_{i} E+R^{T} \Phi_{i} S^{T}\right)+\left(P_{i} E+R^{T} \Phi_{i} S^{T}\right)^{T} A_{i}+E^{T} \mathscr{P}_{\mathscr{K}}^{i} E \\
& +E^{T}\left[\hat{\lambda}_{i i} P_{i}+\left(-\hat{\lambda}_{i i}-\lambda_{\mathscr{K}}^{i}\right) \sum_{j \in S_{\ell \mathscr{K}}^{i}, j \neq i} \frac{\hat{\lambda}_{i j}}{-\hat{\lambda}_{i i}-\lambda_{\mathscr{K}}^{i}} P_{j}\right] E .
\end{aligned}
$$

Likewise, since we have $0 \leq \hat{\lambda}_{i j} /\left(-\hat{\lambda}_{i i}-\lambda_{\mathscr{K}}^{i}\right) \leq 1$ and $\sum_{j \in S_{\mathscr{S} K}^{i}, j \neq i} \hat{\lambda}_{i j} /\left(-\hat{\lambda}_{i i}-\lambda_{\mathscr{K}}^{i}\right)=1$, we know that

$$
\begin{aligned}
\Theta_{i}=\sum_{j \in S_{\ell \mathscr{K}}^{i}, j \neq i} \frac{\hat{\lambda}_{i j}-\hat{\lambda}_{i i}-\lambda_{\mathscr{K}}^{i}}{} & {\left[A_{i}^{T}\left(P_{i} E+R^{T} \Phi_{i} S^{T}\right)\right.} \\
& +\left(P_{i} E+R^{T} \Phi_{i} S^{T}\right)^{T} A_{i}+E^{T} \mathscr{P}_{\mathscr{K}}^{i} E \\
& \left.+E^{T}\left(\widehat{\lambda}_{i i} P_{i}-\widehat{\lambda}_{i i} P_{j}-\lambda_{\mathscr{K}}^{i} P_{j}\right) E\right]
\end{aligned}
$$

which means that $\Theta_{i}<0$ is equivalent to $\forall j \in S_{\mathscr{U} \mathscr{K}}, j \neq i$,

$$
\begin{aligned}
A_{i}^{T} & \left(P_{i} E+R^{T} \Phi_{i} S^{T}\right)+\left(P_{i} E+R^{T} \Phi_{i} S^{T}\right)^{T} A_{i} \\
& +E^{T} \mathscr{P}_{\mathscr{K}}^{i} E+E^{T}\left(\widehat{\lambda}_{i i} P_{i}-\widehat{\lambda}_{i i} P_{j}-\lambda_{\mathscr{K}}^{i} P_{j}\right) E<0 .
\end{aligned}
$$

As $\widehat{\lambda}_{i i}$ is lower bounded by $\lambda_{d}^{i}$, we have

$$
\lambda_{d}^{i} \leq \hat{\lambda}_{i i}<-\lambda_{\mathscr{K}}^{i}
$$

which implies that

$$
\lambda_{d}^{i} \leq \widehat{\lambda}_{i i}<-\lambda_{\mathscr{K}}^{i}+\epsilon
$$

for some $\epsilon<0$ arbitrarily small. Then $\hat{\lambda}_{i i}$ can be further written as a convex combination

$$
\hat{\lambda}_{i i}=-\alpha \lambda_{\mathscr{K}}^{i}+\alpha \epsilon+(1-\alpha) \lambda_{d}^{i}
$$

where $\alpha$ takes value arbitrarily in $[0,1]$. Thus, (14) holds if and only if $\forall j \in S_{\mathscr{U} \mathscr{K}}^{i}, i \neq j$,

$$
\begin{aligned}
A_{i}^{T} & \left(P_{i} E+R^{T} \Phi_{i} S^{T}\right)+\left(P_{i} E+R^{T} \Phi_{i} S^{T}\right)^{T} A_{i} \\
& +E^{T} \mathscr{P}_{\mathscr{K}}^{i} E+E^{T}\left(-\lambda_{\mathscr{K}}^{i} P_{i}+\epsilon\left(P_{i}-P_{j}\right)\right) E<0, \\
A_{i}^{T} & \left(P_{i} E+R^{T} \Phi_{i} S^{T}\right)+\left(P_{i} E+R^{T} \Phi_{i} S^{T}\right)^{T} A_{i} \\
& +E^{T} \mathscr{P}_{\mathscr{K}}^{i} E+E^{T}\left(\lambda_{d}^{i} P_{i}-\lambda_{d}^{i} P_{j}-\lambda_{\mathscr{K}}^{i} P_{j}\right) E<0
\end{aligned}
$$

simultaneously hold. Since $\epsilon$ is arbitrarily small, (24) holds if and only if

$$
\begin{aligned}
A_{i}^{T} & \left(P_{i} E+R^{T} \Phi_{i} S^{T}\right)+\left(P_{i} E+R^{T} \Phi_{i} S^{T}\right)^{T} A_{i} \\
+ & E^{T} \mathscr{P}_{\mathscr{K}}^{i} E-\lambda_{\mathscr{K}}^{i} E^{T} P_{i} E<0,
\end{aligned}
$$

which is the case in (25) when $j=i, \forall j \in S_{\mathscr{U} \mathscr{K}}^{i}$. Hence (20) is equivalent to (15).

Therefore, we can conclude that the unforced system (1) with unknown elements in the TRM is stochastically admissible if and only if (14) and (15) hold for $i \in S_{\mathscr{K}}^{i}$ and $i \in S_{\mathcal{K} \mathscr{K}}^{i}$, respectively.

Remark 5. Theorem 4 presents a new necessary and sufficient condition of stochastic admissibility criterion for the MJSS (1). The approach adopted in Theorem 4, which uses the TRM property (the sum of each row is zero), has extended the result of Theorem 1 in [12] to the MJSS. Note that the lower bound, $\lambda_{d}^{i}$, of $\lambda_{i i}$ is allowed to be arbitrarily negative.

Now let us consider the stabilization problem of system (1) in the presence of unknown elements in the TRM. The following theorem presents a condition for the existence of a mode-dependent stabilizing controller of the form in (11).

Theorem 6. Let $\varepsilon_{i}$ be given scalars. Consider the closed-loop system (12) with partially unknown transition probabilities. If there exist matrices $\bar{P}_{i} \in \mathbb{R}^{n \times n}>0$ and nonsingular matrices $\bar{\Phi}_{i} \in \mathbb{R}^{(n-r) \times(n-r)}$, matrices $L_{i} \in \mathbb{R}^{n \times m}$ and $H_{i} \in \mathbb{R}^{m \times(n-r)}$ such that, for each $i \in S$, the following LMIs hold:

$$
\left[\begin{array}{ccc}
A_{i} Y_{i}+Y_{i}^{T} A_{i}^{T}+W_{i}+\lambda_{i i}\left(\varepsilon_{i} E Y_{i}+\varepsilon_{i} Y_{i}^{T} E^{T}-\varepsilon_{i}^{2} E \bar{P}_{i} E^{T}\right) & Y_{i}^{T} F_{i}^{T}(E) & \sqrt{-\lambda_{\mathscr{K}}^{i}} Y_{i}^{T} E_{R} \\
* & -X_{i}(\bar{P}) & 0 \\
* & * & -E_{R}^{T} \bar{P}_{j} E_{R}
\end{array}\right]<0 \text {, }
$$

$$
\forall j \in S_{\mathscr{U} \mathscr{K}}^{i}, \text { if } i \in S_{\mathscr{K}}^{i}
$$




$$
\left[\begin{array}{ccc}
A_{i} Y_{i}+Y_{i}^{T} A_{i}^{T}+W_{i}+\lambda_{d}^{i}\left(\varepsilon_{i} E Y_{i}+\varepsilon_{i} Y_{i}^{T} E^{T}-\varepsilon_{i}^{2} E \bar{P}_{i} E^{T}\right) & Y_{i}^{T} F_{i}^{T}(E) & \sqrt{-\lambda_{d}^{i}-\lambda_{\mathscr{K}}^{i}} Y_{i}^{T} E_{R} \\
* & -X_{i}(\bar{P}) & 0 \\
* & * & -E_{R}^{T} \bar{P}_{j} E_{R}
\end{array}\right]<0,
$$

$$
\forall j \in S_{\mathscr{U} \mathscr{K}}^{i}, \text { if } i \in S_{\mathscr{U} \mathscr{K}}^{i} \text {, }
$$

where

$$
\begin{aligned}
Y_{i} & =\bar{P}_{i} E^{T}+R \bar{\Phi}_{i} S \\
W_{i} & =B_{i}\left(L_{i} E^{T}+H_{i} R\right)+\left(L_{i} E^{T}+H_{i} R\right)^{T} B_{i}^{T} \\
F_{i}(E) & =\left[\sqrt{\lambda_{i \mathscr{K}_{1}}} E_{R}, \ldots, \sqrt{\lambda_{i \mathscr{K}_{m}^{i}}} E_{R}\right]^{T}, \quad \mathscr{K}_{m}^{i} \neq i \\
X_{i}(\bar{P}) & =\operatorname{diag}\left\{E_{R}^{T} \bar{P}_{\mathscr{K}_{1}} E_{R}, \ldots, E_{R}^{T} \bar{P}_{\mathscr{K}_{m}^{i}} E_{R}\right\}, \quad \mathscr{K}_{m}^{i} \neq i .
\end{aligned}
$$

Then there exists a mode-dependent stabilizing controller of the form in (11) such that the closed-loop system is stochastically admissible. The gain of the stabilizing state feedback controller is given by

$$
K_{i}=\left(L_{i} E^{T}+H_{i} R\right)\left(\bar{P}_{i} E^{T}+R \bar{\Phi}_{i} S\right)^{-1}
$$

Proof. Consider the closed-loop system (12) and replace $A_{i}$ by $A_{i}+B_{i} K_{i}$ in (14) and (15), respectively. Then, if $i \in S_{\mathscr{K}}^{i}$, by Schur complement and performing a congruence transformation to (14) by $\left[\begin{array}{cc}Y_{i}^{T} & 0 \\ 0 & I\end{array}\right]$, with $Y_{i}=\left(P_{i} E+S^{T} \Phi_{i} R^{T}\right)^{-1}=$ $\bar{P}_{i} E^{T}+R \bar{\Phi}_{i} S$, we can obtain

$$
\left[\begin{array}{ccc}
A_{i} Y_{i}+Y_{i}^{T} A_{i}^{T}+B_{i} K_{i} Y_{i}+Y_{i}^{T} K_{i}^{T} B_{i}^{T}+\lambda_{i i} Y_{i}^{T} E_{R}\left(E_{R}^{T} \bar{P}_{i} E_{R}\right)^{-1} E_{R}^{T} Y_{i} Y_{i}^{T} F_{i}^{T}(E) & \sqrt{-\lambda_{\mathscr{K}}^{i}} Y_{i}^{T} E_{R} \\
* & -X_{i}(\bar{P}) & 0 \\
* & * & -E_{R}^{T} \bar{P}_{j} E_{R}
\end{array}\right]<0 .
$$

Let $L_{i}=K_{i} \bar{P}_{i}$ and $H_{i}=K_{i} S \bar{\Phi}_{i}$; we have

$$
K_{i}=\left(L_{i} E^{T}+H_{i} R\right) Y_{i}^{-1}=\left(L_{i} E^{T}+H_{i} R\right)\left(\bar{P}_{i} E^{T}+R \bar{\Phi}_{i} S\right)^{-1} .
$$

$$
\begin{aligned}
B_{i} K_{i} Y_{i}+Y_{i}^{T} K_{i}^{T} B_{i}^{T}= & B_{i}\left(L_{i} E^{T}+H_{i} R\right) \\
& +\left(L_{i} E^{T}+H_{i} R\right)^{T} B_{i}^{T}=W_{i},
\end{aligned}
$$

So (31) becomes

$$
\left[\begin{array}{ccc}
A_{i} Y_{i}+Y_{i}^{T} A_{i}^{T}+W_{i}+\lambda_{i i} Y_{i}^{T} E_{R}\left(E_{R}^{T} \bar{P}_{i} E_{R}\right)^{-1} E_{R}^{T} Y_{i} & Y_{i}^{T} F_{i}^{T}(E) & \sqrt{-\lambda_{\mathscr{K}}^{i}} Y_{i}^{T} E_{R} \\
* & -X_{i}(\bar{P}) & 0 \\
* & * & -E_{R}^{T} \bar{P}_{j} E_{R}
\end{array}\right]<0 .
$$

Considering the nonlinear term in the above inequalities, the following inequalities are introduced. For any scalars $\varepsilon_{i}$, $i \in S$, by Lemma 2 , the following inequalities hold:

$$
\begin{aligned}
0 \leq & \left.Y_{i}^{T} E_{R}-\varepsilon_{i} E_{L}\left(E_{R}^{T} \bar{P}_{i} E_{R}\right)\right]\left(E_{R}^{T} \bar{P}_{i} E_{R}\right)^{-1} \\
& \times\left[Y_{i}^{T} E_{R}-\varepsilon_{i} E_{L}\left(E_{R}^{T} \bar{P}_{i} E_{R}\right)\right]^{T} \\
= & Y_{i}^{T} E_{R}\left(E_{R}^{T} \bar{P}_{i} E_{R}\right)^{-1} E_{R}^{T} Y_{i}-\varepsilon_{i} E Y_{i}-\varepsilon_{i} Y_{i}^{T} E^{T}+\varepsilon_{i}^{2} E \bar{P}_{i} E^{T} .
\end{aligned}
$$

Note that $\lambda_{i i} \leq 0$; we have

$$
\begin{aligned}
& \lambda_{i i} Y_{i}^{T} E_{R}\left(E_{R}^{T} \bar{P}_{i} E_{R}\right)^{-1} E_{R}^{T} Y_{i} \\
& \quad \leq \lambda_{i i}\left(\varepsilon_{i} E Y_{i}+\varepsilon_{i} Y_{i}^{T} E^{T}-\varepsilon_{i}^{2} E \bar{P}_{i} E^{T}\right) .
\end{aligned}
$$

So (33) holds if (27) is fulfilled. In a similar way, if $i \in S_{\mathscr{Q} \mathscr{K}}^{i}$, (28) can be worked out from (15). Therefore, the closed-loop system is stochastically admissible, and the desired controller gain is given by (30).

Remark 7. It should be pointed out that if the diagonal elements in the TRM contain unknown ones, the system 
Table 1

\begin{tabular}{lcccc}
\hline Mode & 1 & 2 & 3 & 4 \\
\hline 1 & -1.2 & $\widehat{\lambda}_{12}$ & $\widehat{\lambda}_{13}$ & 0.6 \\
2 & 0.3 & -0.8 & 0.1 & 0.4 \\
3 & $\widehat{\lambda}_{31}$ & $\widehat{\lambda}_{32}$ & -0.6 & 0.3 \\
4 & $\hat{\lambda}_{41}$ & $\widehat{\lambda}_{42}$ & $\widehat{\lambda}_{43}$ & -0.9 \\
\hline
\end{tabular}

admissibility, the existence of the admissible controller, and the controller gains solution will be dependent on $\lambda_{d}^{i}$. The conditions of Theorem 6 are strict LMIs; hence they can be easily tractable by Matlab LMI toolbox.

\section{Examples}

Example 1. Consider system (1) with four operation modes and the following system matrices:

$$
\begin{array}{cc}
E=\left[\begin{array}{ccc}
4 & 0 & 0 \\
0 & 0.8 & 0 \\
0 & 0 & 0
\end{array}\right], \quad E_{L}=\left[\begin{array}{cc}
2 & 0 \\
0 & 0.4 \\
0 & 0
\end{array}\right], \\
E_{R}=\left[\begin{array}{ll}
2 & 0 \\
0 & 2 \\
0 & 0
\end{array}\right], \quad R=\left[\begin{array}{lll}
0 & 0 & 2
\end{array}\right], \quad S=\left[\begin{array}{l}
0 \\
0 \\
1
\end{array}\right], \\
A_{1}=\left[\begin{array}{ccc}
2 & -7 & 1 \\
-5 & -2 & -1 \\
2 & 4 & -5
\end{array}\right], \quad A_{2}=\left[\begin{array}{lll}
5 & 3 & 7 \\
7 & 9 & 3 \\
2 & 4 & 5
\end{array}\right], \\
A_{3}=\left[\begin{array}{ccc}
2 & -5 & 4 \\
-1 & -3 & 3 \\
4 & -6 & 8
\end{array}\right], & A_{4}=\left[\begin{array}{lll}
1 & 4 & 3 \\
2 & 4 & 1 \\
6 & 1 & 4
\end{array}\right], \\
B_{1}=\left[\begin{array}{cc}
0 & 6 \\
-7 & 9 \\
1 & 0
\end{array}\right], & B_{2}=\left[\begin{array}{ll}
5 & 2 \\
0 & 5 \\
6 & 0
\end{array}\right], \\
B_{3}=\left[\begin{array}{cc}
3 & 5 \\
0 & 4 \\
2 & 0
\end{array}\right], & B_{4}=\left[\begin{array}{ll}
0 & 4 \\
7 & 6 \\
3 & 0
\end{array}\right] .
\end{array}
$$

The transition rate matrix is given as shown in Table 1 .

Let $\varepsilon_{1}=1.2, \varepsilon_{2}=-1, \varepsilon_{3}=-0.2, \varepsilon_{4}=2$, and $\hat{\lambda}_{i j}$ denote the unknown elements. Using Theorem 6 and the LMI control toolbox of Matlab, we obtain the controller gains for the system as follows:

$$
\begin{aligned}
& K_{1}=\left[\begin{array}{lll}
3.7123 & 3.7708 & 0.0005 \\
2.1986 & 2.2325 & 0.0006
\end{array}\right] \times 10^{4}, \\
& K_{2}=\left[\begin{array}{ccc}
-0.7952 & -3.3671 & -0.0001 \\
1.1211 & 4.7407 & 0.0002
\end{array}\right] \times 10^{4}, \\
& K_{3}=\left[\begin{array}{lll}
2.5210 & 1.2413 & -0.0000 \\
0.5945 & 0.2927 & -0.0000
\end{array}\right] \times 10^{5}, \\
& K_{4}=\left[\begin{array}{ccc}
5.1907 & -7.2130 & -0.0013 \\
1.7600 & -2.4473 & 0.0008
\end{array}\right] \times 10^{3}
\end{aligned}
$$

Table 2

\begin{tabular}{lccc}
\hline Mode & 1 & 2 & 3 \\
\hline 1 & -1.2 & $\widehat{\lambda}_{12}$ & $\widehat{\lambda}_{13}$ \\
2 & $\widehat{\lambda}_{21}$ & $\widehat{\lambda}_{22}$ & 0.4 \\
3 & 0.3 & 0.5 & -0.8 \\
\hline
\end{tabular}

The closed-loop dynamic responses and the Markovian chain are shown in Figure 1 with the initial condition $x(0)=$ $[0.7,0.5,-2.3]^{T}$.

Example 2. Consider system (1) with three operation modes and the following system matrices:

$$
\begin{gathered}
E=\left[\begin{array}{ll}
2 & 0 \\
0 & 0
\end{array}\right], \quad E_{L}=\left[\begin{array}{l}
2 \\
0
\end{array}\right], \quad E_{R}=\left[\begin{array}{l}
1 \\
0
\end{array}\right], \\
R=\left[\begin{array}{ll}
0 & 1
\end{array}\right], \quad S=\left[\begin{array}{l}
0 \\
2
\end{array}\right], \quad A_{1}=\left[\begin{array}{cc}
1.5 & -1.4 \\
0.1 & 0.2
\end{array}\right], \\
A_{2}=\left[\begin{array}{cc}
-0.5 & -0.3 \\
1 & -1.2
\end{array}\right], \quad A_{3}=\left[\begin{array}{cc}
-0.1 & 0.2 \\
1 & 1
\end{array}\right], \\
B_{1}=\left[\begin{array}{l}
2 \\
0
\end{array}\right], \quad B_{2}=\left[\begin{array}{l}
-1 \\
-3
\end{array}\right], \quad B_{3}=\left[\begin{array}{c}
3 \\
-2
\end{array}\right] .
\end{gathered}
$$

The transition rate matrix is given as shown in Table 2 .

Let $\varepsilon_{1}=1.2, \varepsilon_{2}=-1, \varepsilon_{3}=-0.2, \lambda_{d}^{2}=-1$. In the 2nd row of TRM, the diagonal element $\widehat{\lambda}_{22}$ is unknown; we assign its lower bound $\lambda_{d}^{2}$ a priori with different values $\left(\lambda_{d}^{2} \epsilon\right.$ $(-\infty,-0.4])$. Using Theorem 6 and LMI control toolbox in Matlab, the controller gains for the system are given by

$$
\begin{aligned}
K_{1} & =\left[\begin{array}{ll}
-7.6834 & 0.0014
\end{array}\right] \times 10^{5} \\
K_{2} & =\left[\begin{array}{ll}
-114.1162 & -0.4001
\end{array}\right] \\
K_{3} & =\left[\begin{array}{ll}
529.6195 & 0.5013
\end{array}\right]
\end{aligned}
$$

When $\lambda_{d}^{2}=-2$, we obtain the controller gains differently for the system as follows:

$$
\begin{aligned}
& K_{1}=\left[\begin{array}{ll}
-2.9825 & 0.0003
\end{array}\right] \times 10^{6} \\
& K_{2}=\left[\begin{array}{ll}
504.0862 & -0.4000
\end{array}\right] \\
& K_{3}=\left[\begin{array}{ll}
3.0048 & 0.0005
\end{array}\right] \times 10^{3}
\end{aligned}
$$

It is seen from above that the obtained controller gains are dependent on $\lambda_{d}^{2}$. The closed-loop dynamic responses and the Markovian chain are shown in Figure 2 with the initial condition $x(0)=[0.7,2.89]^{T}$ and $\lambda_{d}^{2}=-1$.

Remark 8. Notice that, in Example 1, all the diagonal elements of TRM are known and, in Example 2, there are unknown diagonal elements in the TRM which illustrate that the controller design is dependent on the lower bound $\lambda_{d}^{i}$ of the corresponding unknown diagonal element. So they cannot be solved by the stabilization criterions developed in [15] which lack considering the case of systems with partly 


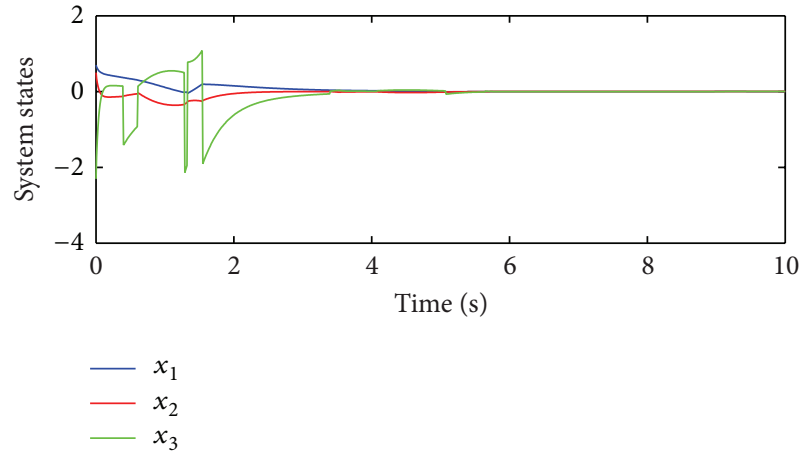

(a)

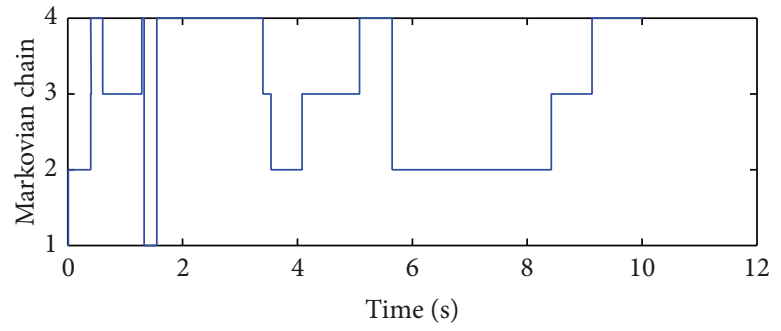

(b)

Figure 1: System states and Markovian chain.

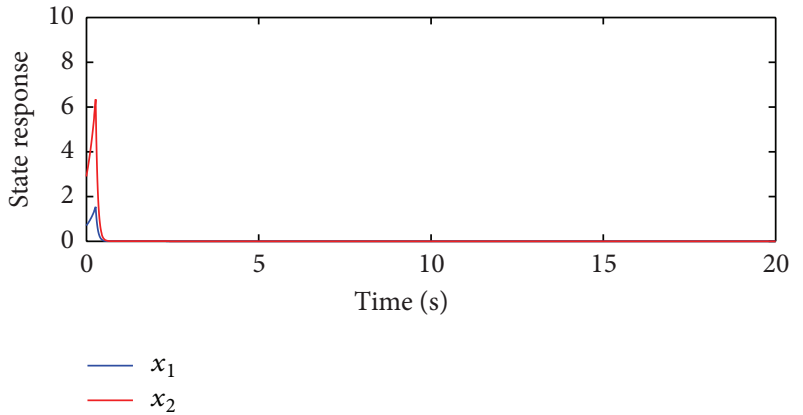

(a)

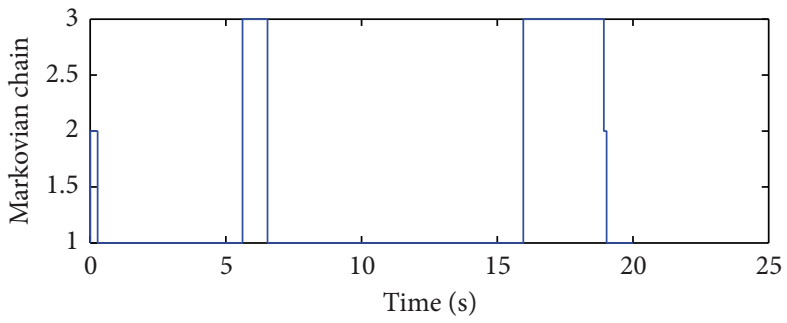

(b)

Figure 2: System states and Markovian chain.

known transition probabilities. Moreover, here examples are for MJSS, while the stabilization criterions developed in [12] which focused on those of normal ones that are special cases of MJSS.

\section{Conclusion}

The problems of stability and state feedback control for continuous-time MJSS with partly known transition probabilities have been studied. A new sufficient and necessary condition for this class of system to be stochastically admissible has been proposed in terms of strict LMIs. Furthermore, sufficient conditions for the state feedback controller are derived, and numerical examples have also been given to illustrate the main results. However, the study of stability and stabilization of continuous-time MJSS with partly known transition probabilities is a basic problem which only serves as a stepping stone to investigate more complicated systems. However, time-delay appears commonly in various practical systems, and researchers have been paying remarkable attention to the problems of analysis and synthesis for timedelay systems [18-24]. The approaches proposed in this paper could be further extended to time-delay systems in our future work. It is expected that the approach can be further used for other analysis and synthesis issues such as $H_{\infty}$ analysis, $H_{\infty}$ synthesis, and other applications such as Markov jumping neural networks with incomplete transition descriptions.

\section{Conflict of Interests}

The authors declare that there is no conflict of interests regarding the publication of this paper.

\section{Acknowledgment}

This work is supported by Postdoctoral Research Sponsorship in Henan Province (Grant no. 2013013).

\section{References}

[1] P. Shi, E.-K. Boukas, and R. K. Agarwal, "Control of Markovian jump discrete-time systems with norm bounded uncertainty and unknown delay," IEEE Transactions on Automatic Control, vol. 44, no. 11, pp. 2139-2144, 1999.

[2] E. K. Boukas and Z. K. Liu, "Robust $H_{\infty}$ control of discrete-time Markovian jump linear systems with mode-dependent timedelays," IEEE Transactions on Automatic Control, vol. 46, no. 12, pp. 1918-1924, 2001.

[3] Y.-Y. Cao and J. Lam, "Robust $H_{\infty}$ control of uncertain Markovian jump systems with time-delay," IEEE Transactions on Automatic Control, vol. 45, no. 1, pp. 77-83, 2000. 
[4] Y. Kang, J. F. Zhang, and S. S. Ge, "Robust output feedback $H_{\infty}$ control of uncertain Markovian jump systems with modedependent time-delays," International Journal of Control, vol. 81, no. 1, pp. 43-61, 2008.

[5] J. Xiong, J. Lam, H. Gao, and D. W. C. Ho, "On robust stabilization of Markovian jump systems with uncertain switching probabilities," Automatica, vol. 41, no. 5, pp. 897-903, 2005.

[6] M. Karan, P. Shi, and C. Y. Kaya, "Transition probability bounds for the stochastic stability robustness of continuousand discrete-time Markovian jump linear systems," Automatica, vol. 42, no. 12, pp. 2159-2168, 2006.

[7] E.-K. Boukas and P. Shi, "Stochastic stability and guaranteed cost control of discrete-time uncertain systems with Markovian jumping parameters," International Journal of Robust and Nonlinear Control, vol. 8, no. 13, pp. 1155-1167, 1998.

[8] L. Zhang and E.-K. Boukas, "Stability and stabilization of Markovian jump linear systems with partly unknown transition probabilities," Automatica, vol. 45, no. 2, pp. 463-468, 2009.

[9] L. Zhang and E.-K. Boukas, " $H_{\infty}$ control for discrete-time Markovian jump linear systems with partly unknown transition probabilities," International Journal of Robust and Nonlinear Control, vol. 19, no. 8, pp. 868-883, 2009.

[10] L. Zhang, E.-K. Boukas, and J. Lam, "Analysis and synthesis of Markov jump linear systems with time-varying delays and partially known transition probabilities," IEEE Transactions on Automatic Control, vol. 53, no. 10, pp. 2458-2464, 2008.

[11] Y. Zhang, Y. He, M. Wu, and J. Zhang, "Stabilization for Markovian jump systems with partial information on transition probability based on free-connection weighting matrices," Automatica, vol. 47, no. 1, pp. 79-84, 2011.

[12] L. Zhang and J. Lam, "Necessary and sufficient conditions for analysis and synthesis of Markov jump linear systems with incomplete transition descriptions," IEEE Transactions on Automatic Control, vol. 55, no. 7, pp. 1695-1701, 2010.

[13] S. Ma, E.-K. Boukas, and Y. Chinniah, "Stability and stabilization of discrete-time singular Markov jump systems with timevarying delay," International Journal of Robust and Nonlinear Control, vol. 20, no. 5, pp. 531-543, 2010.

[14] Z. Wu, H. Su, and J. Chu, "Output feedback stabilization for discrete singular systems with random abrupt changes," International Journal of Robust and Nonlinear Control, vol. 20, no. 17, pp. 1945-1957, 2010.

[15] Y. Xia, E.-K. Boukas, P. Shi, and J. Zhang, "Stability and stabilization of continuous-time singular hybrid systems," Automatica, vol. 45, no. 6, pp. 1504-1509, 2009.

[16] Y. Xia, J. Zhang, and E.-K. Boukas, "Control of discrete singular hybrid systems," Automatica, vol. 44, no. 10, pp. 2635-2641, 2008.

[17] S. Xu and J. Lam, Robust Control And Filtering of Singular Systems, vol. 332, Springer, Berlin, Germany, 2006.

[18] E. Uezato and M. Ikeda, "Strict LMI conditions for stability, robust stabilization, and Ho control of descriptor systems," in Proceedings of the 38th IEEE Conference on Decision and Control (CDC '99), pp. 4092-4097, December 1999.

[19] P. Liu, "Improved delay-dependent robust exponential stabilization criteria for uncertain time-varying delay singular systems," International Journal of Innovative Computing, Information and Control, vol. 9, no. 1, pp. 165-178, 2013.

[20] L. Wu, W. Zheng, and H. Gao, "Dissipativity-based sliding mode control of switched stochastic systems," IEEE Transactions on Automatic Control, vol. 58, no. 3, pp. 785-791, 2013.
[21] X. Su, P. Shi, L. Wu et al., "A novel control design on discretetime Takagi-Sugeno fuzzy systems with time-varying delays," IEEE Transactions on Fuzzy Systems, vol. 21, no. 4, pp. 655-671, 2013.

[22] K. S. Park and J. T. Lim, "Exponential stability of singularly perturbed discrete systems with time-delay," International Journal of Innovative Computing, Information and Control, vol. 9, no. 2, pp. 865-874, 2013.

[23] L. Wu, X. Su, and P. Shi, "Sliding mode control with bounded $L_{2}$ gain performance of Markovian jump singular time-delay systems," Automatica, vol. 48, no. 8, pp. 1929-1933, 2012.

[24] L. Wu, X. Su, P. Shi, and J. Qiu, "A new approach to stability analysis and stabilization of discrete-time T-S fuzzy timevarying delay systems," IEEE Transactions on Systems, Man, and Cybernetics B: Cybernetics, vol. 41, no. 1, pp. 273-286, 2011. 


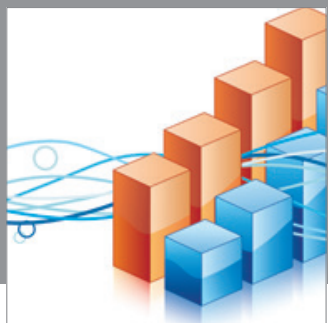

Advances in

Operations Research

mansans

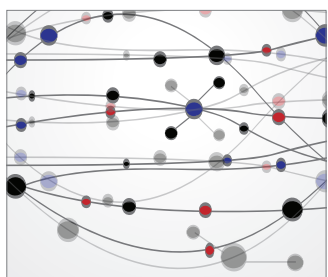

The Scientific World Journal
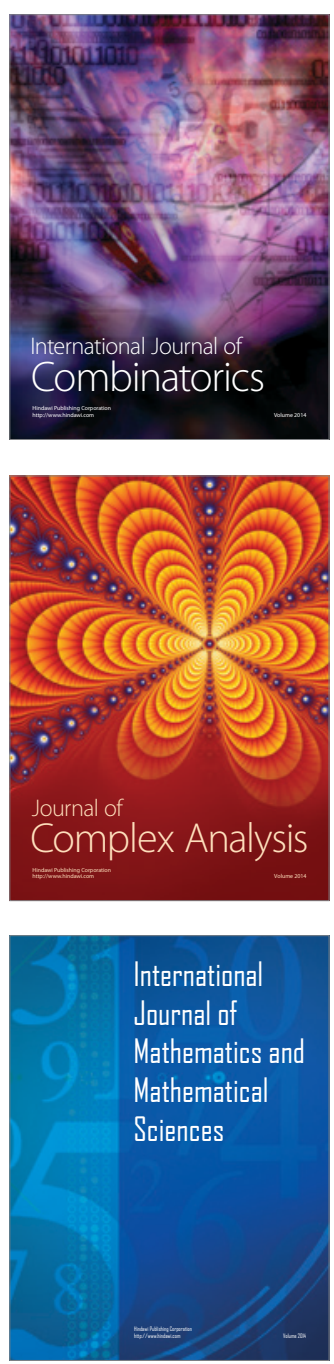
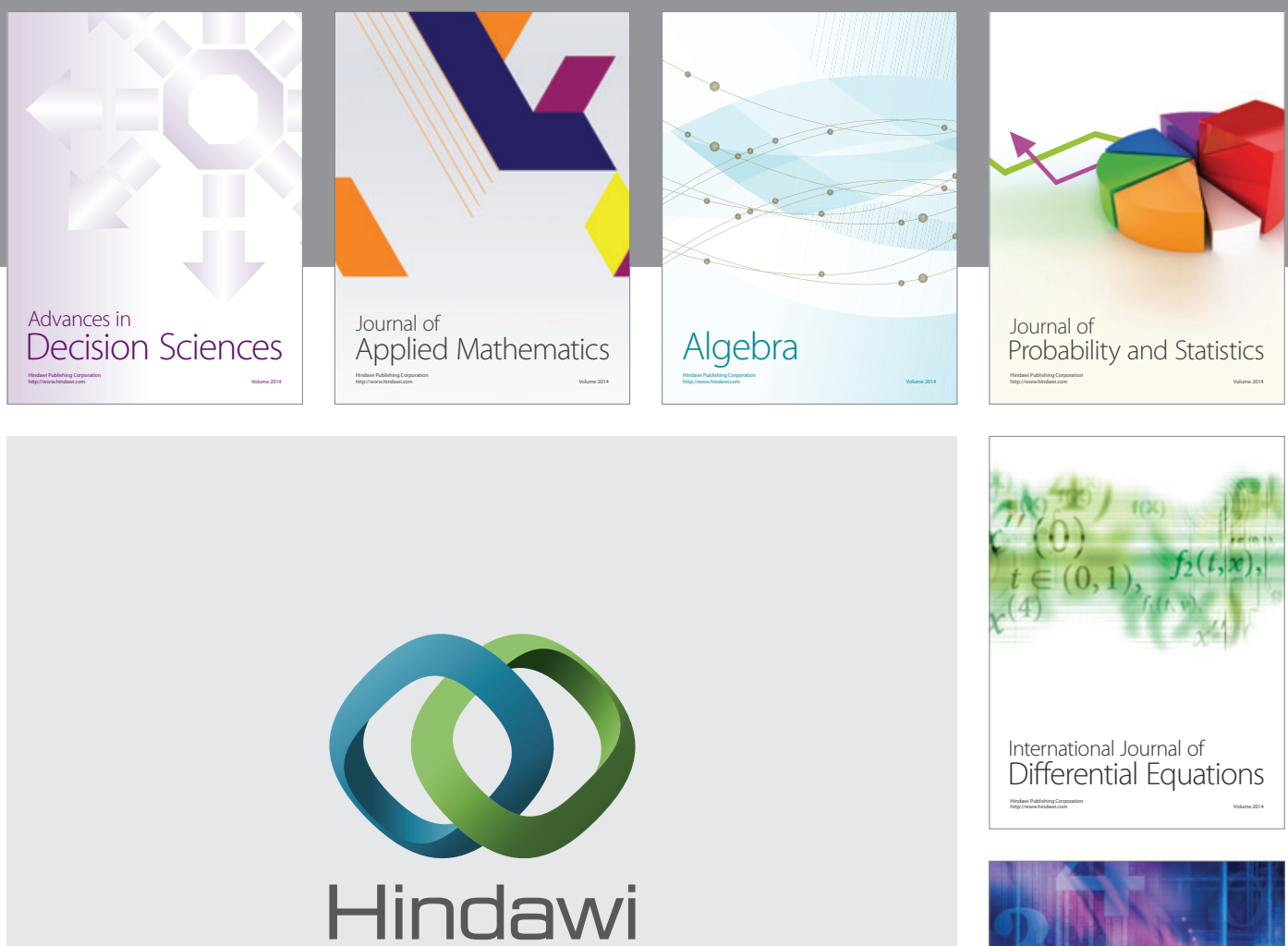

Submit your manuscripts at http://www.hindawi.com
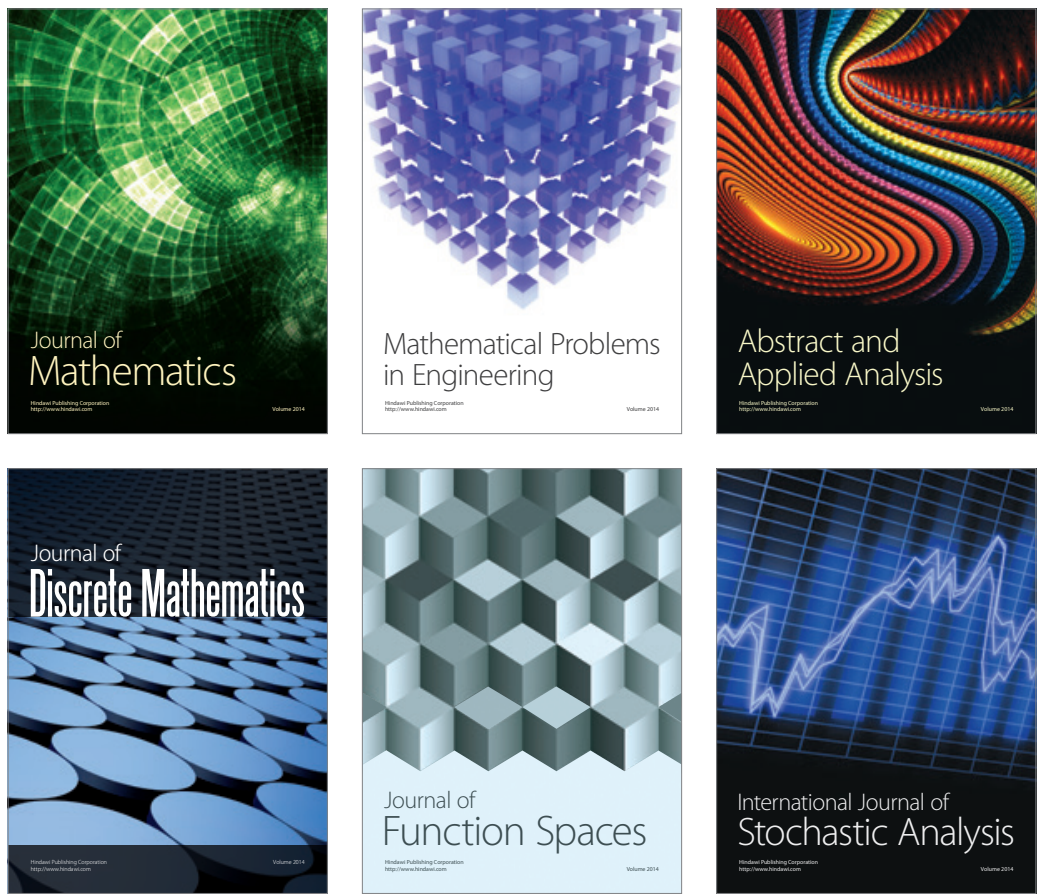

Journal of

Function Spaces

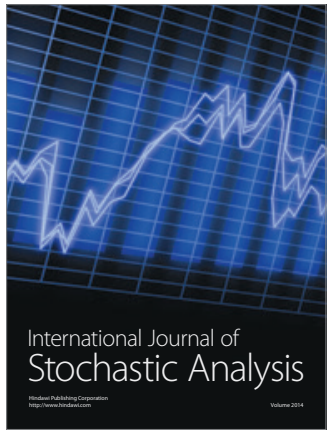

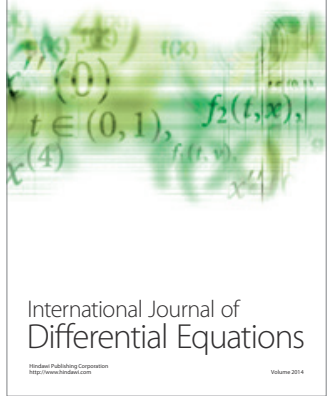
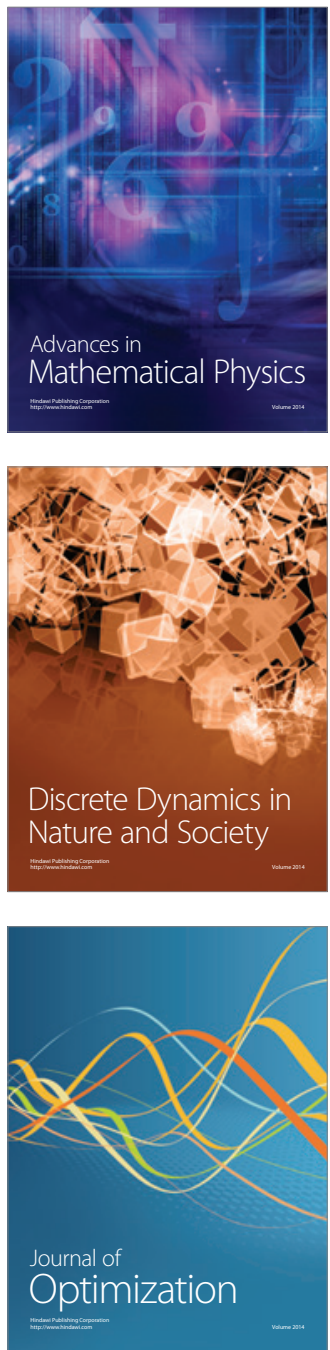